Pacific Journal of Mathematic 


\section{CLASSES OF RINGS TORSION-FREE OVER THEIR CENTERS}

\section{LOUIS ROWEN}

Let $J($ ) denote the intersection of the maximals ideals of a ring. The following properties are studied, for a ring $R$ torsion-free over its center $C$ : $(i) J(R) \cap C=J(C)$; (ii) "Going up" from prime ideals of $C$ to prime ideals of $R$; (iii) If $M$ is a maximal ideal of $R$ then $M \cap C$ is a maximal ideal of $C$; (iv) if $M$ is a maximal (resp. prime) ideal of $C$, then $M=M R \cap C$. Properties (i)-(iv) are known to hold for many classes of rings, including rings integral over their centers or finite modules over their centers. However, using an idea of Cauchon, we show that each of (i)-(iv) has a counterexample in the class of prime Noetherian PI-rings.

Let $R$ be a ring with center $C$. Throughout this note, we assume that $R$ is torsion-free as $C$-module, i.e., $r c \neq 0$ for all nonzero $r$ in $R, c$ in $C$. (In particular, this is the case if $R$ is prime.) Let $J(R)=\cap$ \{maximal ideals of $R$ \}.

$R$ is a $P I$-ring if there exists a noncommutative polynomial $f\left(X_{1}, \cdots, X_{m}\right)$ with coefficients \pm 1 , such that $f\left(r_{1}, \cdots, r_{m}\right)=0$ for all $r_{i}$ in $R$. The basic facts about $P I$-rings are in [6, Chapter X], as well as in [10]. Kaplansky's theorem implies that if $R$ is a $P I$-ring, then $J(R)$ is the Jacobson radical of $R$, so clearly $J(R) \cap C \subseteq J(C)$. A natural question is, "Under what conditions does $J(R) \cap C=J(C)$ ?," or, more generally, "Is there any general correspondence between $J(R)$ and $J(C)$ ?" An answer for PI-rings given in [12, Theorem 5.9], is that $J(R)=0$ implies $J(C)=0$. The object of this note is to tie this question in with other notions which often arise (especially in $P I$-theory). Then we give some pathological examples, which show that many interesting negative properties (including $J(R) \cap C \neq J(C)$ ) occur in such natural classes as the class of prime Noetherian PIrings. Some easy theory is developed to cast some light on the sharpness of these counterexamples. (Although the counterexamples are associative, one may note that associativity is not needed in the positive results.)

Call an ideal $A$ of $C$ contracted if $A=A^{\prime} \cap C$ for some ideal $A^{\prime}$ of $R$. (By [11, Theorem 2], semiprime $P I$-rings have a wealth of contracted ideals of the center.)

Lemma 1. An ideal $A$ of $C$ is contracted, iff $A R \cap C \subseteq A$.

Proof. Suppose $A$ is contracted, i.e. $A=A^{\prime} \cap C$. Then $A R \subseteq A^{\prime}$, 
so $A R \cap C \subseteq A^{\prime} \cap C=A$. Conversely, if $A R \cap C \leqq A$, then $A R \cap C=A$, so $A$ is contracted.

Lemma 1 gives us a useful way of characterizing contracted ideals of $C$ and shows that any chain condition on the lattice of ideals of $R$ induces the corresponding condition on the lattice of contracted ideals of $C$. However, it is often hard to apply lemma 1 to determine the precise make-up of \{contracted ideals of $C$ \}. Some specific information can be obtained.

REMARK 2. Every principal ideal of $C$ is contracted.

Proof. We wish to show $c R \cap C \subseteq c C$ for every nonzero $c$ in C. But if $c r \in C$ then $0=[c r, x]=c[r, x]$ for all $x$ in $R$, implying $r \in C$.

REMARK 3. If $C$ is a valuation domain, then every ideal of $C$ is contracted.

Proof. Recall that, given $x$ and $y$ in a valuation domain $C$, either $x$ divides $y$ or $y$ divides $x$. Hence, if $A$ is an ideal of $C$ and if $c=\sum_{i=1}^{t} a_{i} r_{i} \in A R \cap C$, then (by induction on $t$ ) some $a_{j}$ divides every $a_{i}, 1 \leqq i \leqq t$. Write $a_{i}=a_{j} a_{i 1}$. Then

$$
c=a_{j} \sum \alpha_{i 1} r_{i} \in \alpha_{j} R \cap C \subseteq \alpha_{j} C A
$$

(cf. Remark 2). Thus, $A R \cap C \subseteq A$, so $A$ is contracted.

To examine contracted ideals further, we use central localization (cf. [12]), which is briefly described as follows: Given a multiplicatively closed set $S \subseteq C$ containing 1 , let $R_{S}$ be the classical localization (as $C$-module) of $R$ respect to $S ; R_{S} \approx R \otimes_{C} C_{S}$. If $T \subseteq R$, we write $T_{S}$ for $\left\{x s^{-1} \mid x \in T\right\}$. If $P$ is a prime ideal of $C$, then we write $R_{P}$ for $R_{C-P}$; note that $C_{P}$ has a unique maximal ideal $P_{P}$. There is a canonical injection $\psi_{s}: R \rightarrow R_{S}$, given by $r \rightarrow r 1^{-1}$, and $C_{S}=\operatorname{Cent}\left(R_{S}\right)$. Moreover, $R_{S}$ is always torsion free over $C_{S}$. If $P$ is a prime ideal of $C$, write $\psi_{P}$ for $\psi_{C-P}$ and note that $\psi_{P}^{-1}$ is a lattice injection of \{prime ideals of $R_{P}$ \} into \{prime ideals of $R$ \}. For $S=C-\{0\}$, call $R_{S}$ the ring of central quotients of $R$.

Lemma 4. (i ) If $A$ is a contracted ideal of $C$, then $A_{S}$ is a contracted ideal of $C_{s}$. (ii) If $B$ is a contracted ideal of $C_{s}$, then $\psi_{s}^{-1}(B)$ is a contracted ideal of $C$.

Proof. (i ) If $c s^{-1} \in C_{S} \cap A_{S} R_{S}$, then, for some $s_{1}$ in $S, c s_{1} \in$ 
$A R \cap C \leqq A$, implying $c s^{-1}=\left(c s_{1}\right)\left(s s_{1}\right)^{-1} \in A_{S}$.

(ii) Suppose $c \in \psi_{s}^{-1}(B) R \cap C$. Then $c 1^{-1} \in B R_{S} \cap C_{S} \subseteq B$, so $c \in \psi_{s}^{-1}(B)$.

Proposition 5. If $C$ is Prufer, then every prime ideal of $C$ is contracted.

Proof. Let $P$ be a prime ideal of $C$. Then $C_{P}$ is a valuation domain, so $P_{P}$ is contracted (by Remark 3). But $P$ is then contracted, by Lemma 4 (ii).

Of course, if every prime ideal of a ring is contracted, then every semiprime ideal of the ring is contracted. Another property of interest is "going up". We say that $R$ satisfies $G U\left(P, P_{1}\right)$ if, for every prime ideal $P^{\prime}$ of $R$ with $P=P^{\prime} \cap C$, there exists a prime ideal $P_{1}^{\prime} \supseteq P^{\prime}$, with $P_{1}=P_{1}^{\prime} \cap C . \quad G U\left(P, P_{1}\right)$ occurs to some extent in every prime $P I$-ring (cf. [12, Theorem 4.16]); letting $G U$ denote $G U\left(P, P_{1}\right)$ for all prime ideals $P \subseteq P_{1}$ of $C$, it is natural to ask under what conditions $R$ satisfies $G U$.

All the ideas discussed so far can be related through central localization, as follows:

Proposition 6. Let $\mathscr{R}$ be a class of rings, such that, if $R \in \mathscr{R}$ and $P$ is any prime ideal of $R$, then $R_{P} \in \mathscr{R}$. Consider the following sentences:

(i) $J(C)=J(R) \cap C$ for all $R$ in $\mathscr{R}$.

(ii) $J(C) \subseteq J(R)$ for all $R$ in $\mathscr{R}$.

(iii) $G U$ for all $R$ in $\mathscr{R}$.

(iv) For every $R$ in $\mathscr{R}$, if $P^{\prime}$ is a maximal ideal of $R$, then $P^{\prime} \cap C$ is maximal in $C$.

(v) For every $R$ in $\mathscr{R}$, each maximal ideal of $C$ is contracted.

(vi) For every $R$ in $\mathscr{R}$, each prime ideal of $C$ is contracted. We have (i) $\Leftrightarrow$ (ii) $\Leftrightarrow$ (iii) $\Leftrightarrow$ (iv) $\Rightarrow$ (v) $\Leftrightarrow$ (vi).

Proof. (i) $\Rightarrow$ (ii). Trivial.

(ii) $\Rightarrow$ (iii). Let $P_{1} \subseteq P$ be prime ideals $C$, with $P_{1}=P_{1}^{\prime} \cap C$. Take a maximal ideal $B$ of $R_{P}$ containing $\left(P_{1}^{\prime}\right)_{P}$. Then

$$
P_{P}=J\left(C_{P}\right) \subseteq J\left(R_{P}\right) \subseteq B,
$$

so $P=\psi_{P}^{-1}(B) \cap C$; letting $P^{\prime}=\psi_{P}^{-1}(B) \supseteqq P_{1}^{\prime}$, shows that $G U\left(P_{1}, P\right)$ holds.

(iii) $\Rightarrow$ (iv) Clear.

(iv) $\Rightarrow(\mathrm{v})$. Let $P$ be a prime ideal of $C$. Then $P_{P}$ is the only 
maximal ideal of $C_{P}$. Thus, for any maximal ideal $B$ of $R_{P}, P_{P}=B \cap C_{P}$, by (iv), implying $P=\psi_{P}^{-1}(B) \cap C$.

$(\mathrm{v}) \Rightarrow(\mathrm{vi})$. Immediate; localize at the given prime.

$(\mathrm{vi}) \Rightarrow(\mathrm{v})$. Trivial.

(iv) and (v) $\Rightarrow(\mathrm{i}) . \quad J(C)=\cap\{$ maximal ideals of $C\}=C \cap(\cap\{$ maximal ideals of $R\})=C \cap J(R)$.

For the rest of this note, (i)-(vi) refer to the sentences given in Proposition 6. Sentences (v) and (vi) do not imply (i)-(iv), as evidenced by an example (Bergman-Small $[1, \S 1]$ ) of a prime PI-ring whose center is a valuation domain, but which does not satisfy $G U$. Hence, by Remark 3, we have (vi), but (iii) fails (and thus (i)-(iv) fail in various central localizations). The following remarks are easy and well known.

REMARK 7. The usual proof of the Cohen-Seidenberg theorem can be modified to show that any integral extension of an integral domain satisfies $G U$. (This fact was observed in [2] and extended in [13].) Since "torsion-free over $C$ " implies $C$ is a domain, we see that $\{R$ integral over $C\}$ satisfies (i)-(vi).

REMARK 8. If $R$ is finitely spanned as a $C$-module then $R$ is integral over $C$, of bounded degree. This is is seen via [8, p. 238 and p. 335]. Hence, any ring of this form satisfies (i)-(iv). (R. Snider showed me a proof of (ii) even in the non-torsion-free case.)

REMARK 9. If $R$ has a unique maximal ideal, then $C$ is local and (i), (ii), (iv), and (v) hold. Indeed, let $M$ be the maximal ideal of $R$. For any noninvertible element $c$ in $C$, clearly $c C \subseteq M$. Thus, \{nonunits of $C$ \} is the unique maximal ideal of $C$, equal to $M \cap C$, so (i), (ii), (iv), and (v) follow easily. (Of course this class of rings is not closed under central localization.)

There is also the following general situation where $(v)$ holds:

Proposition 10. (i) Every prime ideal $P$ of $C$, minimal over a contracted ideal $A$ of $C$, is contracted. (ii) Every minimal prime ideal of $C$ is contracted.

Proof. ( i ) \{ideals $\widetilde{B} \supseteqq A R \mid \widetilde{B} \cap C \cong P\}$ is nonempty, and this has a maximal element $\widetilde{P}$, which is clearly prime. Since $\widetilde{P} \cap C$ is prime in $C$ and $A \subseteq \widetilde{P} \cap C \subseteq P$, we have $P=\widetilde{P} \cap C$.

(ii) Every minimal prime ideal of $C$ is minimal over a suitable principal ideal, which is contracted (by Remark 2). 
Hence, any prime ring whose center has Krull Dimension 1 (no two nonzero primes are comparable) satisfies $G U$, so (i)-(vi) hold in this instance. An example of such a ring is the free noncommutative algebra over a commutative domain of Krull Dimension 1.

Having seen some situations in which some all of the sentences in Proposition 6 hold, we shall now look for counterexamples to (v). Example 11(b) will be "generic" in flavor, whereas Example 13 will be Noetherian. Incidentally, in view of Remark 9, this will indicate one of the complications of noncommutative localization of Noetherian $P I$-rings.

EXAMPLE 11. (a) Let $\xi_{i j}^{(k)}, 1 \leqq i, j \leqq n, k=1,2$, be commutative indeterminates over a field $F$, and let $F(\xi)$ be the field generated by all $\xi_{i j}^{(k)}$ over $F$. Let $T$ be the $n \times n$ matrix ring $M_{n}(F(\xi))$, with matric units $\left\{e_{i j} \mid 1 \leqq i, j \leqq n\right\}$, and let $X_{k}$ be the "generic" matrix $\sum_{i j} \xi_{i j}^{(k)} e_{i j}$. The ring $R_{0}$ generated by $F, X_{1}$, and $X_{2}$, is the famous "ring of generic matrices," and, by a theorem of Small, $R_{0}$ satisfies $G U$. Moreover, every central localization of $R_{0}$ satisfies $G U$ (and thus (i)-(vi)), by [12, Theorem 4.24]. In fact, this class can be expanded to \{rings whose central kernel is a maximal ideal of the center\}, cf. [12, Theorem 4.24]. This example makes the following example quite surprising:

(b) Notation as in (a), let $X=X_{1}$, and let $\mu_{1}, \cdots, \mu_{n}$ be the characteristic values of $X^{-1}$. Define $\alpha_{1}=\sum_{i=1}^{n} \mu_{i}, \alpha_{2}=\sum_{i<j} \mu_{i} \mu_{j}, \cdots$, $\alpha_{n}=\mu_{1} \mu_{2} \cdots \mu_{n}$. We claim that $R$, the subring of $T$ generated by $R_{0}$ and $\alpha_{1}, \cdots, \alpha_{n}$, is a counterexample to (v).

Let $C=\operatorname{Cent}(R)$ and let $A=\sum \alpha_{i} C$. Clearly $A R=R$ (since $\left.\sum_{i=1}^{n}(-1)^{i-1} \alpha_{i} X^{i}=1\right)$. We will prove the claim by showing $A \neq C$. The starting point is Procesi's observation that the characteristic values of $X$ are algebraically independent (seen by specializing all $\xi_{i j}^{(1)}$ to 0 for $i \neq j$ ). Hence the $\mu_{i}$ are algebraically independent, and the theory of symmetric polynomials in commutative indeterminates (cf. [8, pp. 133-4]) will be applied to $\alpha_{1}, \cdots, \alpha_{n}$.

Let $C_{1}=F\left[\alpha_{1}, \cdots, \alpha_{n}\right]$ and let $D$ be the subring of $R$ generated by $X$ and $C_{1}$. Note that $X^{-1}=\sum_{i=1}^{n}(-1)^{i-1} \alpha_{i} X^{i-1} \in D$. Suppose there are $c_{i}$ in $C$ such that $\sum_{u} \alpha_{u} c_{u}=1$. Specializing all $\xi_{i j}^{(2)}$ to 0 , we may assume that each $c_{u} \in C \cap D$. Since $\alpha_{1}, \cdots, \alpha_{n}$ are algebraically independent, we will have reached a contradiction once we prove that $C \cap D=C_{1}$.

So suppose $c=\sum_{k=q}^{t} f_{l k}(\alpha) X^{k} \in C \cap D$, where each $f_{k}(\alpha) \in C_{1}$. Write $c$ in this form, with $t$ minimal. First we show that $t \leqq 0$. Otherwise, assume $t>0$. Write $r_{1}=\sum_{k=q}^{0} f_{k}(\alpha) X^{k}$. Diagonalizing, we may assume $X^{-1}=\sum_{i=1}^{n} \mu_{i} e_{i i}$. Let $g\left(X^{-1}\right)=\sum_{i=1}^{n}(-1)^{i-n} \alpha_{n-i-1} X^{i-n}$, where $\alpha_{0}=1$. Clearly $g\left(X^{-1}\right)=\alpha_{n} X$, so we can write 


$$
\begin{aligned}
\alpha_{n}^{t} c & =\alpha_{n}^{t} r_{1}+\sum_{k=1}^{t} \alpha_{n}^{t-k} f_{k}(\alpha) g\left(X^{-1}\right)^{k} \\
& =\alpha_{n}\left(\alpha_{n}^{t-1} r_{1}+\sum_{k=1}^{t-1} \alpha_{n}^{t-k-1} f_{k}(\alpha) g\left(X^{-1}\right)^{k}\right)+f_{t}(\alpha) g\left(X^{-1}\right)^{t},
\end{aligned}
$$

a matrix with entries in $F\left[\mu_{1}, \cdots, \mu_{n}\right]$, a polynomial ring. Now $g\left(X^{-1}\right)^{t} e_{j j}=\left(\mu_{1} \cdots \mu_{j-1} \mu_{j+1} \cdots \mu_{n}\right)^{t} e_{j j}$. Examining the entry in the $j, j$ position, for $i \neq j$, we see that $\mu_{i}$ divides both $\alpha_{n}$ and $f_{t}(\alpha) g\left(X^{-1}\right)^{t}$, implying $\mu_{i}$ divides $\alpha_{n}^{t} c$. By symmetry, $\mu_{1} \cdots \mu_{n} \mid \alpha_{n}^{t} c$; reversing steps shows that $\mu_{j} \mid f_{t}(\alpha)\left(\mu_{1} \cdots \mu_{j-1} \mu_{j+1} \cdots \mu_{n}\right)^{t}$. Hence $\mu_{j} \mid f_{t}(\alpha)$ for each $j$; By symmetry, $f_{t}(\alpha)=\alpha_{n} h$ for some element $h$ in $F\left[\mu_{1}, \cdots, \mu_{n}\right]$.

Since $h$ is symmetric in $\mu_{1}, \cdots, \mu_{n}, h$ is in $D_{1}$; hence, we can write $c=\sum_{k=q}^{t-2} f_{k}(\alpha) X^{k}+\left(f_{t-1}(\alpha)+h g\left(X^{-1}\right)\right) X^{t-1}$, contrary to the choice of $t$ minimal. Thus, $t \leqq 0$, after all.

In other words, $c$ is a polynomial in $X^{-1}$ and the $\alpha_{i}$. Write $c=\sum_{i=1}^{n} f\left(\mu_{1}, \cdots, \mu_{n}\right) e_{i i}$. Switching $\mu_{i}$ and $\mu_{j}$ merely interchanges the (equal) coefficients of $e_{i i}$ and $e_{j j}$, so we see that $f$ is symmetric in the $\mu_{\imath}$. Therefore $c \in C_{1}$, as desired.

Examples $11 \mathrm{a}$ and $11 \mathrm{~b}$ show, in particular, that any of the sentences (i) through (vi) may hold in some prime PI-ring, but fail in a finitely generated central extension. Also, $11 \mathrm{~b}$ is in fact affine, that is, finitely generated (as a ring) over a field. However, \{affine prime $P I$-rings $\}$ is not closed under central localization at prime ideals of the center; in fact, Amitsur proved that all affine prime $P I$-rings are semiprimitive (cf. $[10, \mathrm{p} .102]$ ), so (i) holds in this class.

In view of Remarks 7 and 8, and [5], clearly (i)-(vi) hold for large classes of Noetherian $P I$-rings, and it is natural to ask whether (vi) holds for all prime Noetherian PI-rings. First let us examine the idea of example $11 \mathrm{~b}$. It is well-known that a prime PI-ring can be embedded in a matrix ring over a field. Example 11b "works" because there is a suitably general matrix $(X)$ which is not integral over the center, but for which we have the coefficients of the characteristic polynomial of its inverse. But for Noetherian rings, Schelter proved [13, Theorem 2]: If $R$ is a prime Noetherian PIring then, for any $r$ in $R$, every characteristic value $\alpha$ of $r$ satisfies an equation of the form $\alpha^{t}=\sum_{i=0}^{t-1} \alpha^{2} r_{i}$, for suitable $r_{\imath}$ in $R$.

Thus, if $\alpha^{-1} \in R$ then, multiplying by $\alpha^{1-t}$, we conclude that $\alpha \in R$. In particular, for an element $r$ in an arbitrary prime Noetherian $P I$-ring, if $\operatorname{det}\left(r^{-1}\right) \in R$ then $\operatorname{det}\left(r^{-1}\right)$ is a unit in $R$. Hence, the idea of example $11 \mathrm{~b}$ fails for prime Noetherian PI-rings.

Now we give in an example of a prime, affine Noetherian PIring which does not satisfy $(v)$. Of course, such an example cannot be integral over its center, by Remark 7, and until recently, all 
known prime Noetherian PI-rings were integral (over their centers). Cauchon [3] and Schelter [13] have discovered non-integral, prime Noetherian PI-rings. Although, as can be seen, both examples satisfy (vi), Cauchon's example is representative of a wide class including counterexamples to $(\mathrm{v})$. (Small informed me that, using an approach similar to that of Schelter [13], he has also obtained a counterexample to (v).) Let us start by considering Cauchon's example in its general setting. Recall that a derivation of a ring $R$ is an additive map $D: R \rightarrow R$ satisfying $(x y) D=(x D) y+x(y D)$ for all $x, y$ in $R$.

EXAMPLE 12. Let $L$ be a commutative domain with derivation $D$, and let $e_{11}, e_{12}, e_{21}, e_{22}$ be matric units of $M_{2}(L)$. For any element $a$ in $L$, let $a^{\prime}=a\left(e_{11}+e_{22}\right)+(\alpha D) e_{12} . \quad H=\left\{a^{\prime} \mid a \in L\right\}$ is a commutative ring isomorphic to $L$ (via the map $a \mapsto a^{\prime}$ ). Choose $x$ in $L$, and let $R$ be the subring of $M_{2}(L)$ generated by $H$ and $x M_{2}(L)$. As shown in [3], $R$ is a finitely spanned left (and right) module over $H$, with generators $x e_{i j}, 1 \leqq i, j \leqq 2$. Since the ring of central quotients of $R$ is the (simple) ring of matrices over the field of fractions of $L, R$ is prime. Clearly $H \cap \operatorname{Cent}(R)=\left\{a^{\prime} \mid a D=0\right\}$.

EXAmPle 13. A prime, affine Noetherian $P I$-ring $R$ which does not satisfy $(\mathrm{v})$.

Let $L_{0}$ be the field generated over $\boldsymbol{Q}$ by the indeterminates $x, y_{1}, y_{2}, z_{1}$, and $z_{2}$, and let $L$ be the $Q$-subalgebra of $L_{0}$ generated by $x, y_{1}, y_{2}, z_{1}, z_{2}$, and $\left(1-y_{1} z_{1}\right) z_{2}^{-1}$. Let $L_{1}=\boldsymbol{Q}\left[x, z_{1}\right]\left(z_{2}\right)$, and we extend the zero derivation on $L_{1}$ to a derivation $D$ on $L_{1}\left[y_{1}, y_{2}\right]$ via the conditions $y_{1} D=y_{2} z_{2}$ and $y_{2} D=y_{2}^{2}$. By restriction, $D$ is also a derivation on $L$.

We claim $L \cap L_{1}=\{g \in L \mid g D=0\}$. Indeed, suppose $g D=0$ and $g=\sum_{i=0}^{t} f_{i}\left(y_{2}\right) y_{1}^{i}$ for suitable $f_{i}\left(y_{2}\right)$ in $L_{1}\left[y_{2}\right]$, chosen such that $t$ is minimal. The coefficient of $y_{1}^{t}$ in $g D$ is $\left(f_{t}\left(y_{2}\right)\right) D$, which is thus 0 ; it follows easily that $f_{t}\left(y_{2}\right)$ equals some element $\mu$ in $L_{1}$. If $t>0$, then the coefficient of $y_{1}^{t-1}$ in $g D$ is $\left(f_{t-1}\left(y_{2}\right) D+t \mu y_{2} z_{2}\right)=0$; hence $\mu=0$, contrary to the minimality of $t$. Therefore $t=0$, and $g=\mu \in L_{1}$, proving the claim.

Now let $R$ be built from $L$, using the construction and notation of Example 12. Since $L$ is Noetherian and $R$ is a finite $L$-module, $R$ is left and right Noetherian. Also, $R$ is clearly affine, as well as prime (cf. Example 12). We claim that $R$ does not satisfy (v). Indeed, with $C=$ Cent $R$, let $A=z_{1}^{\prime} C+z_{2}^{\prime} C$. Since

$$
1=z_{1}^{\prime} y_{1}^{\prime}+z_{2}^{\prime}\left(\left(1-y_{1} z_{1}\right) z_{2}^{-1}\right)^{\prime} \in A R,
$$

it suffices to show that $A \neq C$. Suppose to the contrary that 
$z_{1}^{\prime} c_{1}+z_{2}^{\prime} c_{2}=1$, for suitable $c_{\imath}$ in $C$. Taking the parts of degree 0 in $x$, we may assume $c_{1}, c_{2} \in H$. Then we can write $c_{i}=d_{i}^{\prime}$ for suitable $d_{i}$ in $L$. By Example $12, d_{i} D=0$, so $d_{i} \in L \cap L_{1}$, implying $d_{i} \in \boldsymbol{Q}\left[z_{1}\right]\left(z_{2}\right)$. Now $z_{1} d_{1}+z_{2} d_{2}=1$, which we assert is an impossibility. Well, taking homogeneous components in terms of $z_{2}$, we may assume that $d_{1}=h_{1}\left(z_{1}\right)$ and $d_{2}=h_{2}\left(z_{1}\right) z_{2}^{-1}$ for suitable $h_{i}\left(z_{1}\right)$ in $\boldsymbol{Q}\left[z_{1}\right]$. Since $d_{2} \in L$, it follows that $d_{2}=\left(\left(1-y_{1} z_{1}\right) z_{2}^{-1}\right) d$ for some element $d$ in $L$. Viewing $d_{2}$ as a polynomial in $y_{1}$, with coefficients in $L_{1}$, we see that $d_{2}$ must have degree $\geqq 1$. But this contradicts the fact that $d_{2} \in L_{1}$. We conclude that $A \neq C$, as wanted.

\section{REFERENCES}

1. G. Bergman and L. Small, PI-degree and prime ideals, J. Algebra, 33 (1975), 435-462.

2. W. D. Blair, Right Noetherian rings integral over their centers. J. Algebra, 27 (1973), 189-198.

3. G. Cauchon, Un exemple d'anneau premier, noethérien, à identité polynômiale, to appear.

4. E. Formanek, Central polynomials for matrix rings, J. Algebra, 23 (1972), 129-132.

5. — Noetherian PI-rings, Communications in Algebra, 1 (1974), 79-86.

6. N. Jacobson, Structure of Rings, Amer. Math. Soc. Colloquium Publication XXXVII, Providence, RI. (1964).

7. I. Kaplansky, Commutative Rings (revised ed.,) University of Chicago Press, (1974).

8. Lang, Algebra, Addison-Wesley, (1965).

9. Nagarajan, Groups acting on Noetherian rings, Nieuw. Arch. Wisk., 16 (1968), 25-29.

10. C. Procesi, Rings with Polynomial Identy, Marcel Dekker, (1973).

11. L. Rowen, Some results on the center of a ring with polynomial identity, Bull. Amer. Math Soc., 79 (1973), 219-223.

12. - On rings with central polynomial, J. Algebra, 31 (1974), 393-426.

13. W. Schelter, Integral extensions of rings satisfying a polynomial identity, J. Algebra, (to appear).

Received March 12, 1976

BAR ILAN UNIVERSITY

RAMAT GAN, ISRAEL 


\section{PACIFIC JOURNAL OF MATHEMATICS}

\section{EDITORS}

RICHARD ARENS (Managing Editor)

University of California

Los Angeles, CA 90024

R. A. BEAUMONT

University of Washington

Seattle, WA 98105

C. C. Moore

University of California

Berkeley, CA 94720

\section{J. DugundJI}

Department of Mathematics

University of Southern California

Los Angeles, CA 90007

R. Finn and J. Milgram

Stanford University

Stanford, CA 94305

\section{ASSOCIATE EDITORS}

\section{E. F. BECKENBACH}

B. H. NEUMANN

F. WoLF

K. YOSHIDA

\section{SUPPORTING INSTITUTIONS}

\author{
UNIVERSITY OF BRITISH COLUMBIA \\ CALIFORNIA INSTITUTE OF TECHNOLOGY \\ UNIVERSITY OF CALIFORNIA \\ MONTANA STATE UNIVERSITY \\ UNIVERSITY OF NEVADA \\ NEW MEXICO STATE UNIVERSITY \\ OREGON STATE UNIVERSITY \\ UNIVERSITY OF OREGON \\ OSAKA UNIVERSITY
}

\author{
UNIVERSITY OF SOUTHERN CALIFORNIA \\ STANFORD UNIVERSITY \\ UNIVERSITY OF HAWAII \\ UNIVERSITY OF TOKYO \\ UNIVERSITY OF UTAH \\ WASHINGTON STATE UNIVERSITY \\ UNIVERSITY OF WASHINGTON \\ AMERICAN MATHEMATICAL SOCIETY
}

The Supporting Institutions listed above contribute to the cost of publication of this Journal, but they are not owners or publishers and have no responsibility for its content or policies.

Mathematical papers intended for publication in the Pacific Jaurnal of Mathematics should be in typed form or offset-reproduced, (not dittoed), double spaced with large margins. Please do not use built up fractions in the text of your manuscript. You may however, use them in the displayed equations. Underline Greek letters in red, German in green, and script in blue. The first paragraph or two must be capable of being used separately as a synopsis of the entire paper. Items of the bibliography should not be cited there unless absolutely necessary, in which case they must be identified by author and Journal, rather than by item number. Manuscripts, in triplicate, may be sent to any one of the editors. Please classify according to the scheme of Math. Reviews, Index to Vol. 39. All other communications should be addressed to the managing editor, or Elaine Barth, University of California, Los Angeles, California, 90024.

The Pacific Journal of Mathematics expects the author's institution to pay page charges, and reserves the right to delay publication for nonpayment of charges in case of financial emergency

100 reprints are provided free for each article, only if page charges have been substantially paid. Additional copies may be obtained at cost in multiples of 50 .

The Pacific Journal of Mathematics is issued monthly as of January 1966. Regular subscription rate: $\$ 7200$ a year (6 Vols., 12 issues). Special rate: $\$ 36.00$ a year to individual members of supporting institutions.

Subscriptions, orders for back numbers, and changes of address should be sent to Pacific Journal of Mathematics, 103 Highland Boulevard, Berkeley, California, 94708.

PUBLISHED BY PACIFIC JOURNAL OF MATHEMATICS, A NON-PROFIT CORPORATION

Printed at Kokusai Bunken Insatsusha (International Academic Printing Co., Ltd.). 8-8, 3-chome, Takadanobaba, Shinjuku-ku, Tokyo 160, Japan.

Copyrit (C) 1975 by Pacific Journal of Mathematics Manufactured and first issued in Japan 


\section{Pacific Journal of Mathematics \\ Vol. 69, No. $2 \quad$ June, 1977}

Carol Alf and Thomas Alfonso O'Connor, Unimodality of the Lévy spectral

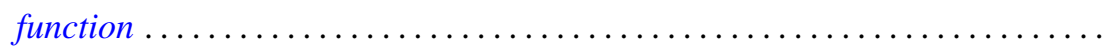

S. J. Bernau and Howard E. Lacey, Bicontractive projections and reordering of

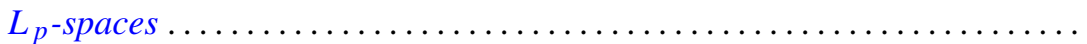

Andrew J. Berner, Products of compact spaces with bi-k and related spaces..... 303

Stephen Richard Bernfeld, The extendability and uniqueness of solutions of ordinary differential equations ...............................

Marilyn Breen, Decompositions for nonclosed planar m-convex sets ..........

Robert F. Brown, Cohomology of homomorphisms of Lie algebras and Lie

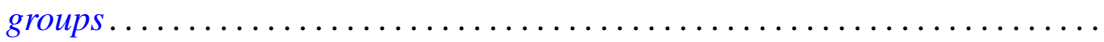

Jack Douglas Bryant and Thomas Francis McCabe, A note on Edelstein's iterative test and spaces of continuous functions ....................

Victor P. Camillo, Modules whose quotients have finite Goldie dimension ....... 333

David Downing and William A. Kirk, A generalization of Caristi's theorem with

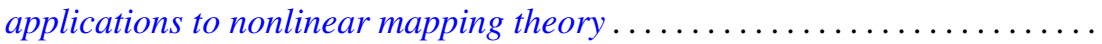

Daniel Reuven Farkas and Robert L. Snider, Noetherian fixed rings ...........

Alessandro Figà-Talamanca, Positive definite functions which vanish at

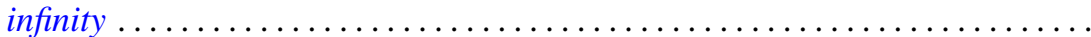

Josip Globevnik, The range of analytic extensions .................. 365

André Goldman, Mesures cylindriques, mesures vectorielles et questions de

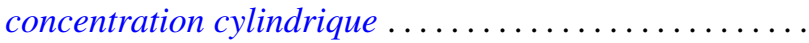

Richard Grassl, Multisectioned partitions of integers..........

Haruo Kitahara and Shinsuke Yorozu, A formula for the normal part of the

Laplace-Beltrami operator on the foliated manifold .... .

Marvin J. Kohn, Summability $R_{r}$ for double series .........

Charles Philip Lanski, Lie ideals and derivations in rings with involution ..

Solomon Leader, A topological characterization of Banach contractions . .

Daniel Francis Xavier O’Reilly, Cobordism classes of fiber bundles . .

James William Pendergrass, The Schur subgroup of the Brauer group . .

Howard Lewis Penn, Inner-outer factorization of functions whose Fourier series

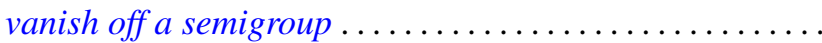

501

William T. Reid, Some results on the Floquet theory for disconjugate definite

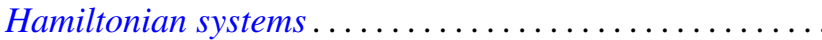

Caroll Vernon Riecke, Complementation in the lattice of convergence

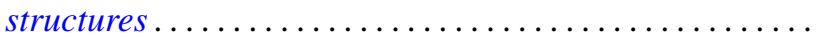

Louis Halle Rowen, Classes of rings torsion-free over their centers ......... 527

Manda Butchi Suryanarayana, A Sobolev space and a Darboux problem ....... 535

Charles Thomas Tucker, II, Riesz homomorphisms and positive linear maps.... 551

William W. Williams, Semigroups with identity on Peano continua ........... 557

Yukinobu Yajima, On spaces which have a closure-preserving cover by finite 